ISSN = 1980-993X - doi:10.4136/1980-993X
www.agro.unitau.br/ambi-agua
E-mail: ambi-agua@agro.unitau.br
Tel.: (12) 3625-4116

\title{
Determinação de trechos de importância em sistemas de distribuição de água usando a vulnerabilidade hidráulica \\ (doi:10.4136/ambi-agua.64)
}

\section{Zacarías Navarro-Roa ${ }^{1}$; Maurício Luiz Sens²; Ramon Lucas Dalsasso ${ }^{3}$; Bruno Segalla Pizzolatti ${ }^{4}$}

\author{
Laboratório de Potabilização de Águas, Departamento de Engenharia Sanitária e Ambiental, \\ Universidade Federal de Santa Catarina \\ Caixa Postal 476, Florianópolis-SC, CEP88040-900, Brasil \\ E-mail: ${ }^{2}$ znavarroa1@hotmail.com; ${ }^{2} \mathrm{mls@ens.ufsc.br;}{ }^{3}$ dalsasso@ens.ufsc.br; ${ }^{4}$ brunosegalla@hotmail.com
}

\section{RESUMO}

Os sistemas de abastecimento de água, por sua configuração, tendem a ser vulneráveis aos perigos internos e externos, que têm o potencial de causar instabilidade na distribuição de água. A avaliação da vulnerabilidade é uma ferramenta que está sendo utilizada para garantir o abastecimento de água em diferentes cenários de falhas, podendo contribuir na busca de alternativas para fornecer água em situações de emergência. $O$ principal objetivo deste trabalho é determinar os trechos importantes ou críticos para um desempenho adequado do sistema de abastecimento de água, quando existem condições emergenciais como, por exemplo, as provocadas por desastres naturais. O desempenho hidráulico do sistema foi avaliado por meio de simulações em EPANET 2.0. A determinação da vulnerabilidade foi realizada por meio de uma aplicação programada em Visual Basic 6.0 e integrada EPANET por meio do módulo ferramenta (Toolkit-EPANET). A vulnerabilidade do sistema foi determinada com a interrupção de um trecho por vez e, desse modo, foi possível calcular, de uma forma dinâmica, a importância dos trechos para o sistema, e comparar esses resultados com o índice de importância relativa por vazão. Os resultados mostraram que a utilização dos índices de Vulnerabilidade-Sistema e Vulnerabilidade-Nó foi mais adequada para estabelecer a importância de um trecho crítico com relação ao desempenho de uma rede.

Palavras-chave: confiabilidade; emergência; sistema de abastecimento de água; vulnerabilidade.

\section{Determination of important pipes segments in water distribution systems using the hydraulic vulnerability}

\begin{abstract}
Water supply systems, due to their configuration, tend to be vulnerable to internal and external threats, which can potentially cause instability in water distribution. The vulnerability assessment is a tool that has been used to ensure the water supply in different failure scenarios, and can assist in seeking alternatives for supplying water in emergency situations. This work aimed mainly at determining the most important or critical segments for an adequate performance of the water supply system when there are emergency conditions such as the ones caused by natural disasters. The system's hydraulic performance was evaluated based on simulations using EPANET 2.0. The vulnerability was determined by using an application written in Visual Basic 6.0 to customize EPANET using its
\end{abstract}


Programmer's Toolkit. The system vulnerability was determined with the interruption of one segment at a time to calculate, in a dynamic way, the importance of each segment to the system, and by comparison of these results to the index of relative importance by flow rate. The results showed that using the indices Vulnerability-System and Vulnerability-Node was adequate to establish the importance of a critical segment in the performance of a network.

Keywords: reliability; emergency; water supply system; vulnerability.

\section{INTRODUÇÃO}

Os sistemas de abastecimento de água por sua extensão e configuração são afetados por problemas técnicos internos e externos, como, por exemplo: as falhas de equipamentos e os perigos naturais, respectivamente. As análises dos danos provocadoos por eventos naturais em diferentes lugares demonstram que os sistemas de abastecimento de água são suscetíveis aos impactos de perigos ambientais (Agardy e Ray, 2001; ALA, 2001).

A avaliacão da vulnerabilidade em sistemas de abastecimento de água está baseada na determinacão da influência de determinados trechos no comportamento de uma rede. Isso é realizado considerando-se a importância dos trechos segundo o diâmetro destes, o qual tem sido a base para a determinacão da importância desses componentes. No entanto, o uso do diâmetro como base do cálculo pode levar a resultados falsos, com relação à importância de um trecho.

O objetivo deste trabalho é determinar os trechos importantes ou críticos para um desempenho adequado do sistema de abastecimento de água, por meio da vulnerabilidade hidráulica do sistema. Para estabelecer a importância dos trechos foram comparadas duas alternativas. A primeira é a sugerida por Goulter e Bouchart (1990), comumente utilizada nas análises de vulnerabilidade em sistemas de abastecimento de água. Já a segunda, é a proposta neste artigo, em que é utilizada a análise hidráulica para definir os trechos de maior influência no desempenho adequado do sistema.

Em zonas com alto risco de desastres ou em grandes cidades é imprescindível a realização da avaliação de vulnerabilidade para garantir um abastecimento adequado nos cenários mais críticos para a distribuição de água. Entre os termos utilizados na avaliaçâo de vulnerabilidade estão o de perigo, o de desastre e o de vulnerabilidade propriamente dito.

Os perigos ambientais são eventos naturais ou antropogênicos que podem provocar danos importantes em sistemas humanos ou ecossistemas. Os desastres acontecem quando os danos provocados pelos perigos alteram o desenvolvimento normal de um sistema, da sociedade ou de ecossistemas. Os desastres estão relacionados com a intensidade dos perigos e com a vulnerabilidade dos sistemas e infra-estruturas, e a vulnerabilidade é a capacidade do sistema de resistir o impacto de um determinado perigo.

$\mathrm{Na}$ projeção de sistemas de abastecimento de água, utiliza-se o conceito de confiabilidade, como medida do desempenho do sistema, em diferentes condições de falhas em um tempo determinado. A confiabilidade é a probabilidade de satisfazer a demanda com pressão adequada para cada nó de consumo em condições anormais de operação (Misirdali, 2003; Ostfeld, 2001; Bao e Mays, 1990; Goulter e Bouchart, 1990). São conhecidos dois tipos de confiabilidade, a mecânica e a hidráulica. Em termos gerais, a vulnerabilidade é o complemento da confiabilidade (Mays, 2004), mas a vulnerabilidade refere-se a perigos e componentes específicos. Da mesma forma que a confiabilidade, existe a vulnerabilidade mecânica e a hidráulica. A vulnerabilidade mecânica corresponde à resistência de um componente ou infra-estrutura frente a determinados perigos. A vulnerabilidade hidráulica ou Vulnerabilidade-Sistema é definida como a probabilidade que o sistema não forneça a vazão total demanda em todos os nós de consumo para o tempo de análise. 
Nos casos de interrupção de um ou vários trechos, as ações corretivas dependem das condições físicas e hidráulicas do sistema e da vazão remanescente que este possa suprir depois do impacto de um fenômeno. Dessa forma, se for possível prever a vulnerabilidade do sistema frente aos perigos a que está exposto, determinando a vazão, a pressão e a qualidade da água que será disponibilizada aos usuários, também será possível prever recursos e ações que permitam satisfazer a demanda em uma situação de emergência.

A determinação do desempenho de uma rede de água é realizada por meio do algoritmo proposto por Gupta e Bhave (1996) e Ostfeld et al., (2002), que permite calcular a confiabilidade (vulnerabilidade hidráulica) quando um trecho está interrompido. A vulnerabilidade é o indicador da importância dos diferentes trechos para o sistema e para os nós. Os resultados são comparados com a proposta de Goulter e Bouchart (1990) para determinar a importância relativa de um trecho.

Goulter e Bouchart (1990) estabelecem a Equação 1 para determinar a importância relativa de um trecho em uma rede de distribuição de água. A Importância Relativa do Trecho $\left(\mathrm{IR}_{\mathrm{L}}\right)$ é a relação entre a vazão no trecho $\left(\mathrm{Q}_{\mathrm{L}}\right)$ e a vazão total da rede $\left(\mathrm{Q}_{\text {total }}\right)$.

$$
\mathrm{IR}_{\mathrm{L}}=\mathrm{Q}_{\mathrm{L}} / \mathrm{Q}_{\text {total }}
$$

A Equação 1 é pouco aplicável em redes malhadas, pois não considera a existência de vias alternativas para abastecimento em um determinado nó, ou seja, a redundância do sistema. Para considerar a redundância é preciso utilizar simulações hidráulicas que permitam comparar a disponibilidade de água nos nós de consumo de uma maneira dinâmica.

Para avaliar o comportamento hidráulico da rede, foi utilizado o software EPANET, o qual foi desenvolvido por Water Supply and Water Resources Division da Agência de Proteção Ambiental dos Estados Unidos de América (USEPA). O EPANET permite realizar simulações hidráulicas em redes de distribuição de água e comparar diferentes cenários de operação do sistema.

No EPANET 2.0, a demanda disponível no nó é satisfeita em todo momento, mas a pressão depende das condições do sistema e da demanda dos nós (Demand-Driven Analyses). Entretanto, a vazão disponível no nó depende da pressão. Por essa razão, em casos de falhas do sistema, é preciso realizar as correções das vazões calculadas por meio do EPANET 2.0. A Equação 2, proposta por Wagner et al. (1988), permite calcular a demanda disponível nos nós, considerando a pressão e a demanda requerida nestes (Wagner et al., 1988; Ostfeld et al., 2002; Wu e Walski, 2006).

$$
\begin{aligned}
& \mathrm{q}_{\mathrm{n}}^{\text {disp }}=0 \text { quando } \mathrm{P}_{\mathrm{n}} \leq 0 \\
& \mathrm{q}_{\mathrm{n}}^{\text {disp }}=\mathrm{q}_{\mathrm{n}}^{\text {req }}\left(\mathrm{P}_{\mathrm{n}} / \mathrm{P}_{\mathrm{n}}^{\text {min }}\right)^{0,5} \text { quando } 0<\mathrm{P}_{\mathrm{n}}<\mathrm{P}_{\mathrm{n}}^{\text {min }} \\
& \mathrm{q}_{\mathrm{n}}^{\text {disp }}=\mathrm{q}_{\mathrm{n}}^{\text {req }} \text { quando } \mathrm{P}_{\mathrm{n}} \geq \mathrm{P}_{\mathrm{n}}^{\min }
\end{aligned}
$$

em que: Pn é a pressão calculada pela simulação em cada estado, $\mathrm{P}_{\mathrm{n}}^{\min }$ é a pressão mínima; $\mathrm{q}_{\mathrm{n}}^{\text {disp }}$ é a demanda disponível e $\mathrm{q}_{\mathrm{n}}^{\text {req }}$ a demanda requerida no nó $\mathrm{n}$.

As Equações 3, 4, 5, 6, 7 e 8 foram propostas por Gupta e Bhave (1996) para determinar a confiabilidade em sistemas de abastecimento de água. Em que, Rnn, é a confiabilidade em nós; ${ }^{\mathrm{q} n, \mathrm{t}}$, é a vazão disponível no nó no instante $\mathrm{t}$; ${ }^{\mathrm{q} n, \mathrm{t}}$ é a vazão demandada no nó para um instante t; $t$ é o instante de simulação; Tno é o total de nós; e Ttotal é o tempo total de simulação. Com a Equação 3 é determinada a confiabilidade nos nós $\left(\operatorname{Rn}_{n}\right)$ e estabelece a relação entre a quantidade de água fornecida e a requerida em cada nó. 


$$
\mathrm{Rn}_{\mathrm{n}}=\sum_{\mathrm{t}=\mathrm{o}}^{\text {Ttotal }} \mathrm{q}_{\mathrm{n}, \mathrm{t}}^{\text {disp }} / \sum_{\mathrm{t}=0}^{\text {Ttotal }} \mathrm{q}_{\mathrm{n}, \mathrm{t}}^{\text {req }}
$$

A Equação 4 determina $\mathrm{Rv}$, que representa o fator de confiabilidade para vazão total no sistema durante o período de análise. Essa é a relação freqüentemente utilizada para definir a confiabilidade e avalia todo o volume movimentado no sistema durante o ciclo de simulação.

$$
\mathrm{Rv}=\sum_{\mathrm{t}=0}^{\text {Ttotal }} \sum_{\mathrm{n}}^{\text {Tno }} \mathrm{q}_{\mathrm{n}, \mathrm{t}}^{\text {disp }} / \sum_{\mathrm{t}=0}^{\text {Ttotal }} \sum_{\mathrm{n}}^{\text {Tno }} \mathrm{q}_{\mathrm{n}, \mathrm{t}}^{\text {req }}
$$

$\mathrm{O}$ déficit de água no tempo é medido com o fator de tempo nó $\left(\mathrm{Ft}_{\mathrm{n}}\right)$, que estabelece a média de tempo e, aproximadamente, $50 \%$ da vazão requerida no nó é considerada satisfatória. A Equação 5 é utilizada para calcular esse fator. Em que $a_{n, t}$ é a disponibilidade aceitável, e $\mathrm{a}_{\mathrm{n}, \mathrm{t}}=1$ se $\mathrm{q}_{\mathrm{n}, \mathrm{t}}^{\text {disp }} / \mathrm{q}_{\mathrm{n}, \mathrm{t}}^{\text {req }} \geq 0,5$ e $\mathrm{a}_{\mathrm{n}, \mathrm{t}}=0$ se $\mathrm{q}_{\mathrm{n}, \mathrm{t}}^{\text {disp }} / \mathrm{q}_{\mathrm{n}, \mathrm{t}}^{\text {req }}<0,5$ (Gupta e Bhave, 1996).

$$
\mathrm{Ft}_{\mathrm{n}}=\left(\sum_{\mathrm{t}=0}^{\text {Ttotal }} \mathrm{a}_{\mathrm{n}, \mathrm{t}}\right) / \text { Ttotal }
$$

O fator de tempo para o sistema (Ft) é a média de tempo em que a vazão para cada nó e cada instante $t$ foi satisfatória. A Equação 6 é utilizada para determinar o fator de tempo para o sistema, em que: Ft é o fator de tempo para o sistema; Tno, é o total de nós; e n, é o nó avaliado no instante $\mathrm{t}$.

$$
\mathrm{Ft}=\sum_{\mathrm{n}}^{\mathrm{Tno}} \mathrm{Ft}_{\mathrm{n}} / \mathrm{Tno}
$$

Gupta e Bhave (1996) consideram o Fator de Nó (Fn), dado pela Equação 7. Em que Tno é o número total de nós, e; $n$, o nó avaliado. Devido a esse fator ser a média geométrica de confiabilidade da vazão para o nó, é sensível à mínima confiabilidade dos nós.

$$
\mathrm{Fn}=\left[\prod_{\mathrm{n}=1}^{\mathrm{Tno}} \mathrm{Rn}_{\mathrm{n}}\right]^{1 / \text { Tno }}
$$

A Equação 8 é utilizada para determinar a confiabilidade hidráulica no sistema (Rnw), com limites de $0 \leq$ Rnw $\leq 1$.

$$
\mathrm{Rnw}=\mathrm{Rv} * \mathrm{Ft} * \mathrm{Fn}
$$

A Vulnerabilidade-Nó ( $\left.\mathrm{Vul}_{\mathrm{no}}\right)$ e a Vulnerabilidade-Sistema $\left(\mathrm{Vul}_{\text {sist }}\right)$ são definidas como o complemento da confiabilidade hidráulica para o nó e para o sistema, respectivamente. Nesse cálculo não é considerado o fator de nó (Fn). O Fn na Equação 8, nos casos em que as condições de falhas provocam falta de água em um nó por todo o tempo da simulação, tem valor de zero. Por essa razão no cálculo da importância de trecho o fator Fn não é considerado.

A importância de um trecho depende da vulnerabilidade que o mesmo provoca quando está interrompido durante a simulação. A Vulnerabilidade-Nó, ou seja, a probabilidade de que o nó $n$ não receba a vazão demandada no tempo de análise, pode ser determinada pela Equação 9. A Equação 10 pode ser utilizada para calcular a Vulnerabilidade-Sistema (Vul sist$_{\text {) }}$.

$$
\begin{aligned}
& \mathrm{Vul}_{\mathrm{no}}=\left(1-\mathrm{Ft}_{\mathrm{n}} * \mathrm{Rn}_{\mathrm{n}}\right) \\
& \mathrm{Vul}_{\mathrm{sist}}=(1-\mathrm{Ft} * \mathrm{Rv})
\end{aligned}
$$




\section{METODOLOGIA}

O algoritmo apresentado na Figura 1, foi utilizado para realizar as análises de vulnerabilidade do sistema. A programação dele foi realizada em Visual Basic 6.0 e integrado ao EPANET 2.0 por meio do módulo Toolkit, proporcionado por EPANET (Rossman, 2000). Os modos de operação-falha e não-falha foram simulados interrompendo um trecho por vez.

A determinação da importância de um trecho está baseada na comparação do funcionamento do sistema em condição de falha (interrupção da vazão do trecho) e em condição normal. Nesse sentido, a pressão mínima $\left(\mathrm{P}_{\mathrm{n}}^{\min }\right)$ é igual á pressão esperada segundo a simulação para condição normal ( $\left.\mathrm{P}_{\mathrm{n}, \mathrm{t}}^{\mathrm{req}}\right)$. Dessa forma, é possível observar o quanto o sistema se desvia da normalidade.

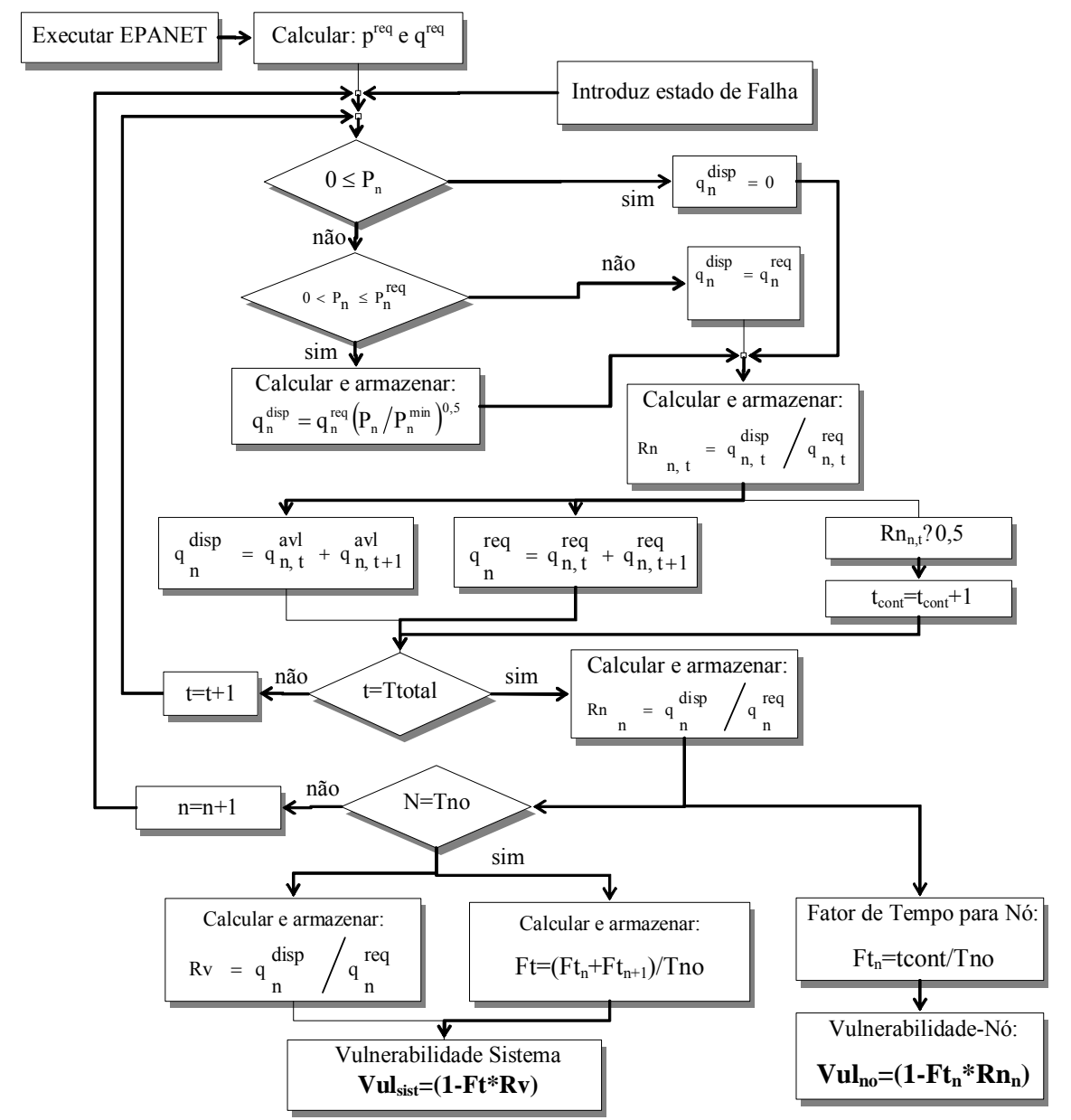

Figura 1. Algoritmo para determinar a vulnerabilidade de sistemas de água (adaptado de Ostfeld et al., 2002; Gupta e Bhave, 1996).

A vazão $\left(\mathrm{q}_{\mathrm{n}, \mathrm{t}}^{\mathrm{req}}\right)$ e a pressão $\left(\mathrm{P}_{\mathrm{n}, \mathrm{t}}^{\mathrm{req}}\right)$ requerida para cada nó em cada tempo foi calculada pela simulação em caso normal. A pressão disponível para cada nó em cada tempo $\left(\mathrm{P}_{\mathrm{n}, \mathrm{t}}^{\mathrm{disp}}\right)$ é determinada na simulação em estado de falha. A vazão disponível foi calculada utilizando-se a Equação 2, a partir da pressão disponível, a pressão requerida e a vazão requerida simuladas para cada hora em EPANET 2.0 com um trecho interrompido. 
Os trechos que provocam maior vulnerabilidade para um nó são os importantes para esse ponto de consumo. Entretanto, os que provocam maior vulnerabilidade para o sistema são os de maior importância para este.

Para determinar a vulnerabilidade de um sistema de água, foi utilizado o exemplo proposto por Rossman (2000), apresentado na Figura 2. Essa rede é simples e apresenta condições não necessariamente reais, no entanto, a configuração permite comparar facilmente os resultados.

\section{RESULTADOS E ANÁLISES}

O estudo avaliou a importância dos trechos para o desempenho do sistema em casos emergenciais. A Figura 2 representa o esquema e dados de entrada do exemplo proposto por Rossman (2000).

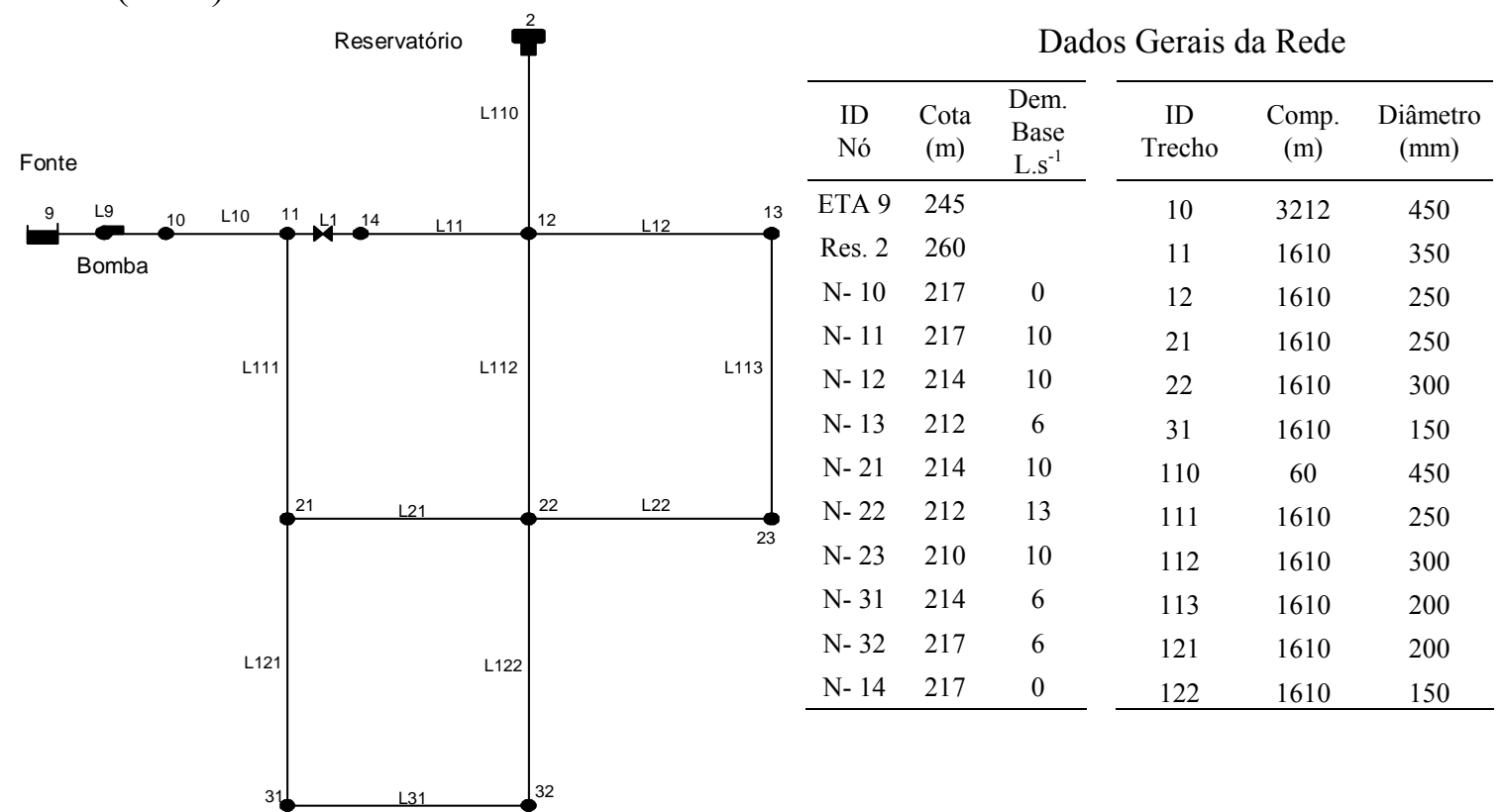

Figura 2. Esquema de rede de distribuição (Extraído de Rossman, 2000).

Na Tabela 1, observa-se o resultado da vulnerabilidade do sistema e dos nós para uma simulação de 5 dias (120 horas). Cada trecho permaneceu interrompido durante o tempo total de simulação. De acordo com a Vulnerabilidade-Sistema ( $\left.\mathrm{Vul}_{\text {sist }}\right)$, os 4 trechos mais importantes são: L-112, L-121, L-111 e L-11 com vulnerabilidade de 2,1\%, 1,9\%, 1,9\% e $0,7 \%$ respectivamente. Segundo IRL, os 4 trechos mais importantes são: L-11, L-111, L-112 e L-12 com importância de $21,5 \%, 10,7 \%, 10,1 \%$ e 5,3\%, no sistema. É preciso esclarecer que o trecho L-10 por ser a adutora era esperado uma alta vulnerabilidade, no entanto, não é considerado na análise de importância dos trechos. 
NAVARRO-ROA, Z.; SENS, M. L.; DALSASSO, R. L.; PIZZOLATTI, B. S. Determinação de trechos de importância em sistemas de distribuição de água usando a vulnerabilidade hidráulica. Ambi-Agua, Taubaté, v. 3, n. 3, p. 95-104, 2008. (doi:10.4136/ambi-agua.64)

Tabela 1. Vulnerabilidade-Nó, Vulnerabilidade-Sistema e $\mathrm{IR}_{\mathrm{L}}$.

\begin{tabular}{|c|c|c|c|c|c|c|c|c|c|c|c|}
\hline \multirow{2}{*}{$\begin{array}{c}\text { ID } \\
\text { Trecho }\end{array}$} & \multicolumn{8}{|c|}{ Vulnerabilidade-Nó $\left(\mathrm{Vul}_{\text {no }}\right)$} & \multirow{2}{*}{$\begin{array}{c}\text { Vul } \\
\text { Sist } \\
\%\end{array}$} & \multirow{2}{*}{$\begin{array}{c}\text { Vazão } \\
\text { Max } \\
\left(L . s^{-1}\right)\end{array}$} & \multirow[t]{2}{*}{$\begin{array}{c}\mathbf{I R}_{\mathbf{L}} \\
\%\end{array}$} \\
\hline & N-11 & $\mathrm{N}-12$ & $\mathrm{~N}-13$ & $\mathrm{~N}-21$ & $\mathrm{~N}-22$ & $\mathrm{~N}-23$ & $\mathrm{~N}-31$ & $\mathrm{~N}-32$ & & & \\
\hline L-10 & 99,80 & 99,80 & 99,80 & 99,80 & 99,80 & 99,80 & 99,80 & 99,80 & 99,80 & 120,08 & 37,20 \\
\hline L-11 & 0,21 & 1,33 & 1,14 & 0,12 & 0,88 & 0,93 & 0,12 & 0,54 & 0,68 & 69,51 & 21,50 \\
\hline L-12 & 0,15 & 0,10 & 1,23 & 0,32 & 0,36 & 0,61 & 0,34 & 0,37 & 0,40 & 16,97 & 5,30 \\
\hline L-21 & 0,18 & 0,38 & 0,43 & 0,03 & 0,51 & 0,49 & 0,06 & 0,21 & 0,30 & 4,86 & 1,50 \\
\hline L-22 & 0,04 & 0,06 & 0,53 & 0,02 & 0,02 & 1,41 & 0,03 & 0,03 & 0,27 & 9,43 & 2,90 \\
\hline L-31 & 0,05 & 0,06 & 0,06 & 0,05 & 0,07 & 0,07 & 0,01 & 1,24 & 0,16 & 3,31 & 1,00 \\
\hline L-110 & 0,00 & 0,00 & 0,00 & 0,00 & 0,00 & 0,00 & 0,00 & 0,00 & 0,00 & 4,08 & 1,30 \\
\hline L-111 & 0,16 & 0,92 & 1,32 & 3,22 & 2,07 & 1,97 & 3,05 & 2,96 & 1,89 & 34,57 & 10,70 \\
\hline L-112 & 1,10 & 0,67 & 1,28 & 2,51 & 2,93 & 2,80 & 2,69 & 2,97 & 2,12 & 32,46 & 10,10 \\
\hline L-113 & 0,09 & 0,05 & 0,03 & 0,14 & 0,15 & 0,20 & 0,15 & 0,17 & 0,12 & 6,57 & 2,00 \\
\hline L-121 & 0,20 & 0,24 & 0,25 & 0,06 & 0,28 & 0,27 & 10,77 & 8,41 & 1,90 & 13,71 & 4,20 \\
\hline L-122 & 0,09 & 0,06 & 0,05 & 0,11 & 0,05 & 0,05 & 1,01 & 3,06 & 0,42 & 7,09 & 2,20 \\
\hline
\end{tabular}

A Figura 3 (a) ilustra o comportamento da pressão e da demanda disponível e requerida para o nó N-32 com a interrupção do trecho L-121 durante o tempo total de simulação. A pressão disponível evidencia uma redução substancial, no momento mais crítico, em que a pressão disponível foi de $57 \mathrm{~m}$, reduzindo a vazão disponível em aproximadamente $15 \%$.

A Figura 3 (b) apresenta o comportamento da vazão e da pressão no nó N-31 quando L121 encontra-se interrompido. Para esse nó, o trecho L-121 se mostra muito importante, a maior Vunerabilidade-Nó ocorre nesse cenário de falha, com aproximadamente $11 \%$. A pressão disponível mínima no nó foi de $52 \mathrm{~m}$, reduzindo a vazão disponível em aproximadamente $20 \%$.

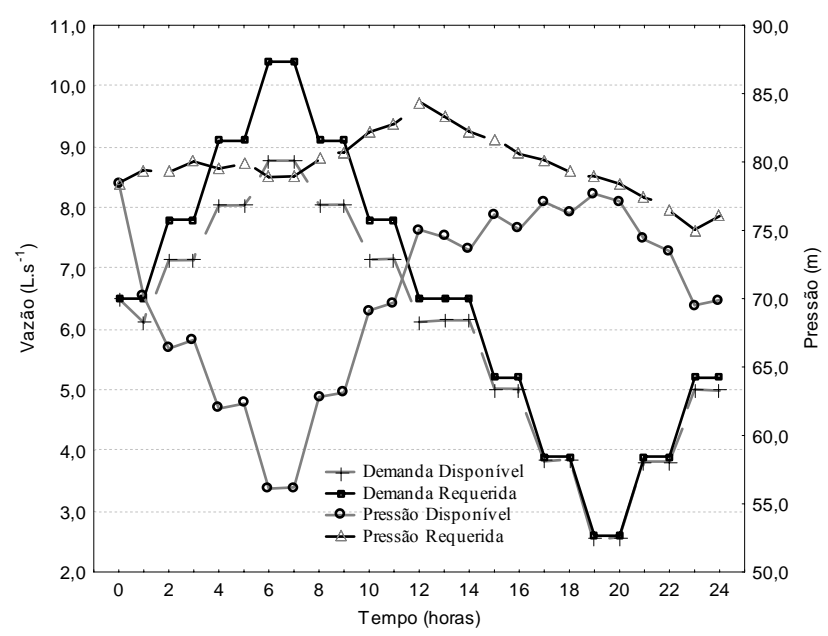

(a)

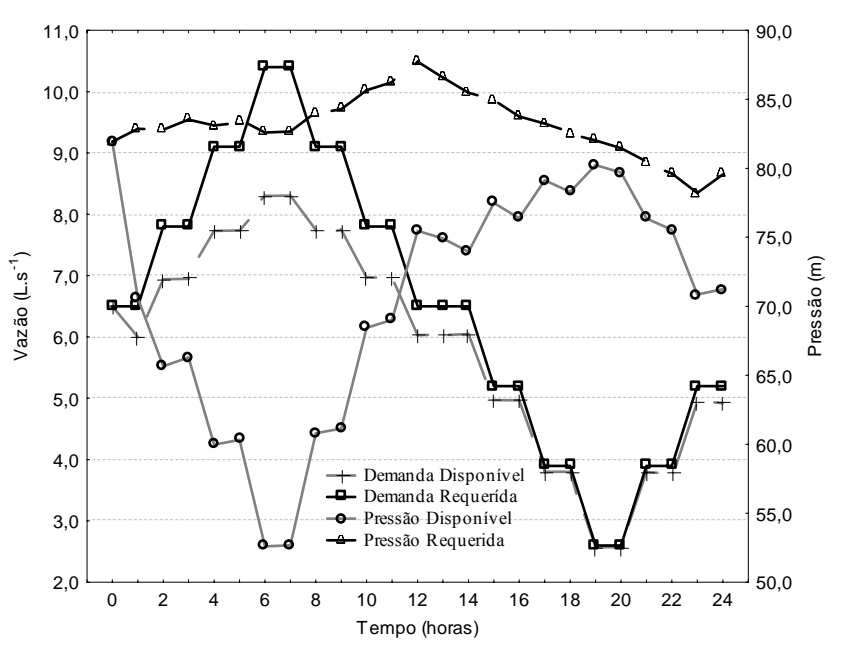

(b)

Figura 3. Comportamento da Demanda e Pressão no nó N-32 (a) e N-31(b) com interrupção do trecho L121.

Na Figura 4, observa-se o comportamento da vulnerabilidade nos nós de consumo com os 4 trechos de maior importância interrompidos. O impacto de L-121 nos nós N-31 e N-32 é o mais evidente; a interrupção dos trechos L-111 e L-112 provocou um desempenho relativamente similar, sendo os nós N-21, N-31 e N-32 os mais afetados. No caso do trecho L11 a vulnerabilidade nos nós foi considerada relativamente baixa, sendo os nós mais afetados o N-12, N-22, N-23 e N-32. 


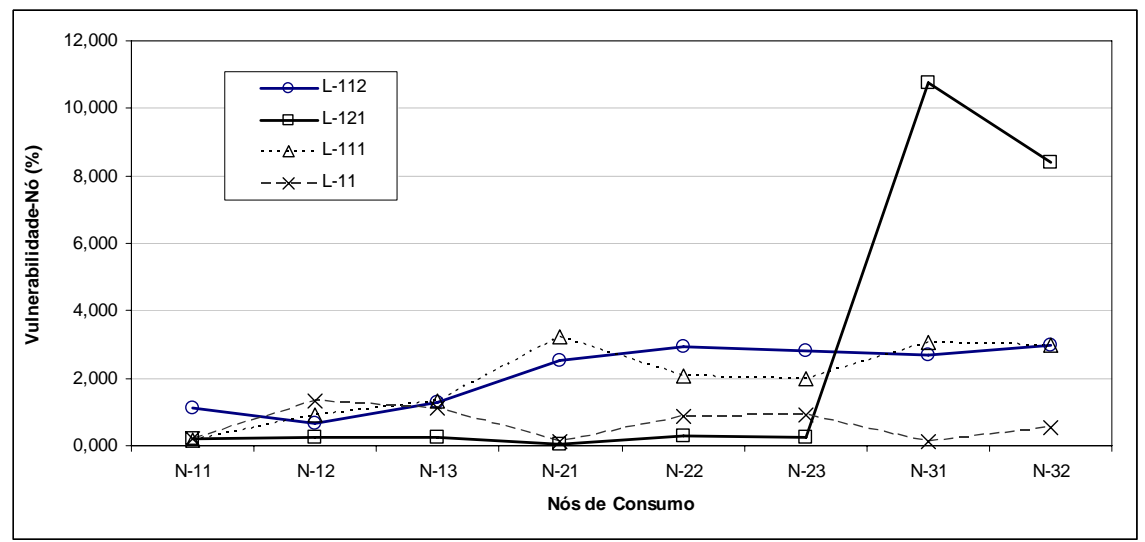

Figura 4. Vulnerabilidade-Nó com interrupção dos trechos L-112, L121, L-111 e L-11.

A Figura 5 permite comparar a importância dos trechos, no que diz respeito à Vulnerabilidade-Sistema e a Importância Relativa de Trecho. Segundo a Vul $\mathrm{Vist}_{\text {st }}$ o trecho considerado mais importante foi o L-112 e relativo ao $\mathrm{IR}_{\mathrm{L}}$, o de maior importância foi o trecho L-11. Para a rede analisada, os resultados apresentaram algumas coincidências. A $\mathrm{Vul}_{\text {Sist }}$ considerou os parâmetros hidráulicos em um determinado tempo, por isso esse índice é mais adequado para determinar a importância dos trechos.

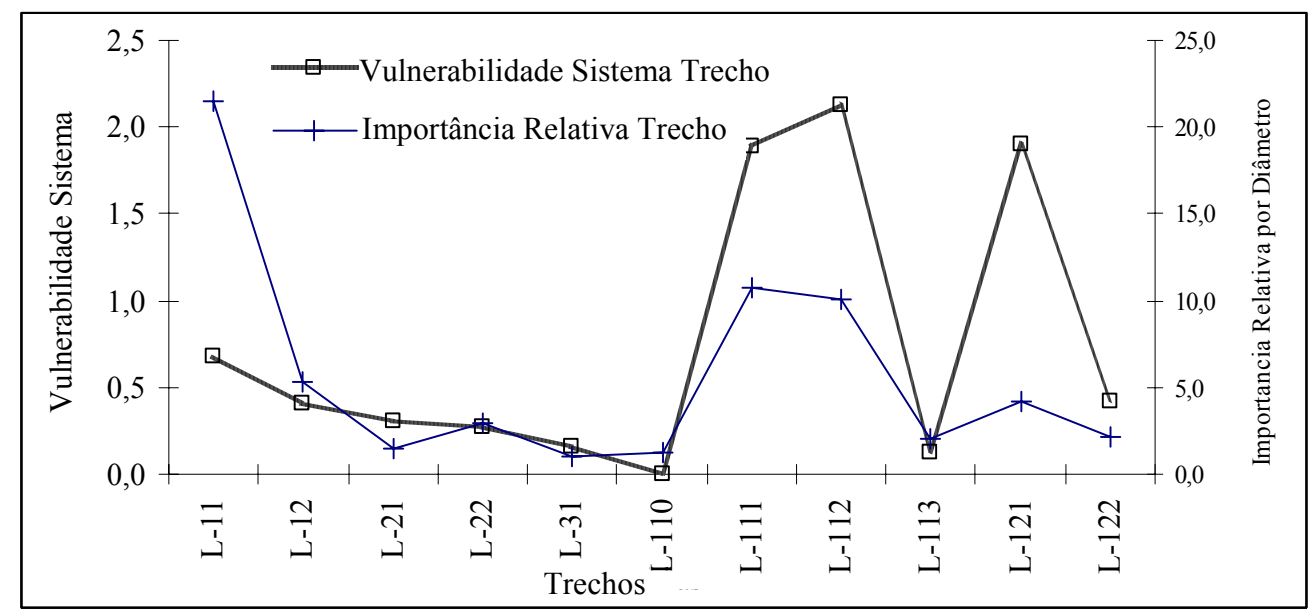

Figura 5. Vulnerabilidade do sistema com trechos interrompidos.

Os resultados demonstraram que a importância de um trecho dentro de uma rede malhada não depende somente da vazão que ela pode fornecer, mas também da configuração da rede, pois esta influencia na importância de cada componente. A aplicação da Vul $1_{\text {Sist }}$ tem maior relevância quando se considera o impacto que o trecho tem sobre um nó particular.

Na Figura 4, observou-se a importância do trecho L-121 para o abastecimento dos nós N31 e N-32. Utilizando a Vulnerabilidade-Nó, identificou-se quais nós foram mais afetados com a interrupção de um trecho. Os resultados obtidos com o cálculo da vulnerabilidade abrangeram a capacidade que o sistema tem de satisfazer a demanda em todos os nós. Por meio de uma análise dinâmica, foi possível ter um resultado mais representativo da realidade que ocorre no sistema, quando um trecho é interrompido por um período de tempo determinado. 
O fato de o trecho L-110 apresentar uma vulnerabilidade nula, não quer dizer que este não é importante para o funcionamento adequado do sistema. Esse resultado significa que, hipoteticamente, se esse componente for fechado, o abastecimento de água pode ser considerado satisfatório. É preciso ressaltar que esta análise não considerou aspectos econômicos, consumo de energia ou controle de pressões, evidenciando a relevância para o trecho L-110.

\section{CONCLUSÃO}

A metodologia permitiu determinar os trechos críticos para o funcionamento normal do sistema. Observou-se uma maior alteração no desempenho da rede, quando um desses trechos críticos é fechado para fins de manutenção ou por alguma situação de emergência provocada por condições extremas.

A determinação do trecho mais crítico para o funcionamento do sistema, sem considerar a adutora, tornou possível observar que, a importância de um trecho não necessariamente tem relação direta com o diâmetro dele. A determinação da importância de trecho, utilizando a vulnerabilidade total do sistema possibilitou uma análise de forma dinâmica, incluindo o abastecimento por trechos alternativos.

Por meio da determinação dos trechos importantes ou críticos, de acordo com a vulnerabilidade provocada por uma interrupção no sistema, obtiveram-se os nós que são mais afetados com o fechamento. Uma análise deste tipo pode ser aplicada na programação de ações que permitam satisfazer a demanda de água em casos de emergências.

A utilização dos índices de Vulnerabilidade-Sistema $\left(\mathrm{Vul}_{\text {sist }}\right)$ e Vulnerabilidade-Nó $\left(\mathrm{Vul}_{\mathrm{no}}\right)$ foi mais adequada para estabelecer a importância de um trecho no que se refere ao desempenho da rede de abastecimento, pois o índice Importância Relativa do Trecho $\left(\mathrm{IR}_{\mathrm{L}}\right)$ não considera a redundância do sistema.

\section{AGRADECIMENTOS}

Os autores agradecem ao programa de bolsa da Organização de Estados Americanos -OEA-LASPAU; ao programa de bolsa da Secretaria de Estado de Educação Superior, Ciência e Tecnologia da República Dominicana; e, à Coordenação de Aperfeiçoamento de Pessoal de Nível Superior do Brasil -CAPES.

\section{REFERÊNCIAS}

AGARDY, F. J.; RAY, A. D. Emergency planning for water utility management (M 19). 3. ed. Denver: Americam Water Works Association, 2001.

AMERICAN LIFELINES ALLIANCE - ALA. Seismic fragility formulations for water systems: part 1. Federal Emergency Management Agency (FEMA) e American Society of Civil Engineers (ASCE). 2001. 96p. Disponível em: <www.americanlifelinesalliance.org>. Acesso em: junho 2008.

BAO, Y.; MAYS, L. Model for water-distribution system reliability. Journal of Hydraulic Engineering, v. 116, n . 9, p. 1119-1137, 1990.

GOULTER, I. C.; BOUCHART, F. Reliability-constrained pipe network model. Journal of Hydraulic Engineering, v. 116, n. 2, p. 211-229, 1990. 
GUPTA, R.; BHAVE, P. Reliability-based of water-distribution systems. Journal of Environmental Engineering, v. 122, n. 1, p. 51-54, 1996.

MAYS, L. W. Vulnerability assessment, emergency response planning: summary of what's available. In: Water supply systems security. New York: McGraw-Hill, 2004. Cap. 3. Disponível em: <www.digitalengineeringlibrary.com>. Accesso em: junho 2006.

MISIRDALI, M. A methodology for calculating hydraulic system reliability of water distribution networks. 2003. 104f. Thesis (Master of Science) - School Of Natural And Applied Sciences, The Middle East Technical University, Ankara, 2003.

OSTFELD, A. Reliability analysis of regional water distribution systems. Urban Water, v. 3 , p. 253-260, 2001.

OSTFELD, A.; KOGAN, D.; SHAMIR, U. Reliability simulation of water distribution systems - single and multiquality. Urban Water, v. 4, p. 53-61, 2002.

ROSSMAN, L. A. EPANET 2: users manual. Cincinnati: U.S. Environmental Protection Agency, 2000.

WAGNER, J.; SHAMIR, U.; MARKS, D. Water distribution reliability: simulation methods. Journal of Water Resources Planning and Management, v. 114, n. 3, p. 276-294, 1988.

WU, Z. Y.; WALSKI, T. M. Pressure dependent hydraulic modelling for water distribution systems under abnormal conditions. In: IWA WORLD WATER CONGRESS, Sept.1014, 2006, Beijing. Proceedings... IWA, 2006. 